\author{
Why you should read this article \\ - To enhance your awareness of the heel as a high-risk area for pressure ulcers \\ - To acknowledge the importance of assessing patients' risk of developing pressure ulcers \\ - To read about techniques and devices that can be used to prevent heel pressure ulcers
}

\title{
Heel pressure ulcers: understanding why they develop and how to prevent them
}

Clare Greenwood

\section{Citation \\ Greenwood C (2021) \\ Heel pressure ulcers: understanding why they develop and how to prevent them. Nursing Standard. doi: 10.7748/ns.2021.e11740}

\section{Peer review}

This article has been subject to external double-blind peer review and checked for plagiarism using automated software

\section{Correspondence} clare.greenwood2@nhs.net y@ClareEGreenwood

Conflict of interest None declared

\section{Accepted \\ 17 May 2021}

\section{Published online}

December 2021

\section{Open access}

This is an open access article distributed under the terms of the Creative Commons Attribution-Non Commercial 4.0 International (CC BY-NC 4.0) licence (see https:// creativecommons.org/licenses/ by-nc/4.0/), which permits others to copy and redistribute in any medium or format, remix, transform and build on this work non-commercially, provided appropriate credit is given and any changes made indicated

\begin{abstract}
Because of its anatomical location and the fact that it has no muscle or fascia and little subcutaneous tissue, the heel is highly vulnerable to pressure damage and pressure ulcer development. Heel pressure ulcers are often severe and challenging to heal. Heel pressure ulcers are caused by factors such as pressure, shear and friction and the vulnerability of the heel to pressure damage is increased by immobility, the patient's skin status, the presence of previous pressure ulcers and/or scar tissue, and suboptimal tissue perfusion. The risk is also increased in patients with diabetes mellitus. The role of nurses includes assessing the risk and preventing the development of heel pressure ulcers. This article discusses the causes of, and risk factors for, heel pressure ulcers and describes techniques and devices that nurses can use to prevent them.
\end{abstract}

\section{Author details}

Clare Greenwood, clinical nurse specialist, tissue viability, St James's University Hospital, Leeds Teaching Hospitals NHS Trust, Leeds, England

\section{Keywords}

clinical, clinical skills, diabetes, diabetic foot ulcers, dressings, leg ulcers, nursing care, pressure area care, pressure ulcers, professional, skin, tissue viability, wound care

\begin{abstract}
Aims and intended learning outcomes
This article aims to enhance nurses' awareness and understanding of the heel as a high-risk area for pressure ulcers, risk factors for developing pressure ulcers, and optimal prevention techniques and devices. The article is based on the author's clinical experience as well as on the available guidance and literature. After reading this article and completing the time out activities you should be able to:

" Explain why the anatomy of the heel makes it particularly vulnerable to pressure damage.

॥ Identify the patient groups that are at increased risk of developing heel pressure ulcers.

॥ Understand the importance of preventing heel pressure ulcers.

॥ Describe prevention techniques and devices.
\end{abstract}

\section{Introduction}

Pressure ulcers, which in the past were also known as bed sores, pressure injuries or decubitus ulcers, have been defined as 'localised damage to the skin and/or underlying tissue, as a result of pressure or pressure in combination with shear', which 'usually occur over a bony prominence but may also be related to a medical device or other object' (European Pressure Ulcer Advisory Panel (EPUAP) et al 2019).

Pressure ulcers can have a significant negative effect on a person's health-related quality of life, including the physical, mental, emotional and social aspects of their everyday life (Spilsbury et al 2007, Gorecki et al 2009). Pain is a significant debilitating symptom in patients who develop a pressure ulcer (Briggs et al 2013, McGinnis et al 2014a). Pressure ulcers can cause serious infections that can lead to osteomyelitis and sepsis, which in turn may lead to multiple organ failure and amputation and can ultimately be fatal (Gorecki et al 2009). Pressure ulcers are a common occurrence and therefore represent a significant financial burden on healthcare services due to the costs of treatment, nursing care and prolonged hospital stays (Guest et al 2015).

Pressure ulcers are categorised according to their depth and severity. The most widely used staging tool categorises pressure ulcers in four categories, from category 1 (intact skin with a localised area of non-blanching erythema) to category 4 (full-thickness skin and tissue loss with exposed 
or directly palpable fascia, muscle, tendon, ligament, cartilage or bone) (EPUAP et al 2019). The staging tool includes two additional categories: unstageable pressure ulcer, where the extent of skin and tissue loss is unknown because it is obscured by slough or necrotic tissue; and suspected deep tissue injury, where deeper damage is suspected because of the presence of persistent non-blanching bruising, a dark wound bed or a blood-filled blister (EPUAP et al 2019).

\section{TIME OUT 1}

Reflect on a patient you have cared for who developed a heel pressure ulcer. Was the category of the pressure ulcer established? Were the potential causes of the heel pressure ulcer investigated? Did it heal and if so, how long did it take?

\section{Significance of the heel as a high-risk area for pressure ulcers}

After the sacrum, the heel is frequently reported as the second most common site for the development of pressure ulcers, as well as the site where the most severe pressure ulcers tend to develop (Greenwood 2020). Heel pressure ulcers can cause pain, reduce mobility and in severe cases result in amputation (Fowler et al 2008). Heel pressure ulcers can be challenging to heal. In a cohort of 140 patients with heel pressure ulcers categorised as category 2 or

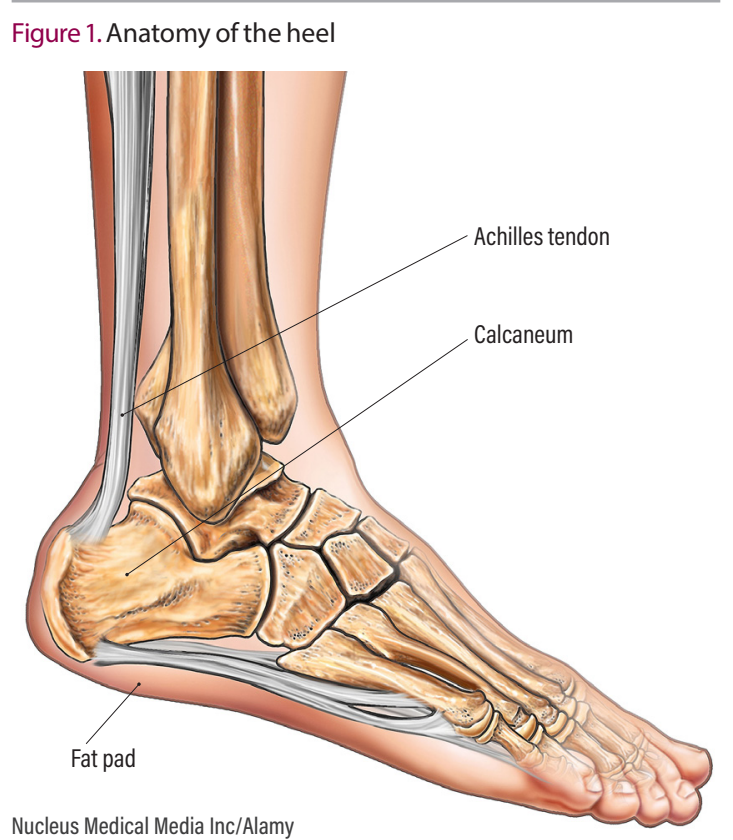

above recruited to a prospective cohort study in one large teaching hospital in the UK, only $42 \%$ were reported to have healed over the 18-month study period (McGinnis et al 2014b).

The anatomy of the heel - shown in Figure 1 - makes it highly vulnerable to pressure damage. The heel is located at the posterior aspect of the foot. It includes the calcaneus (heel bone) and is composed of skin and bone, with no muscle or fascia (connective tissue) and little protective subcutaneous tissue (Davies 2018). The plantar aspect of the heel has a thickened dermis and a large, tough but elastic fat pad, which is designed to absorb the forces from the calcaneus during movement and when the individual is standing upright. The back of the heel has minimal cushioning, with no muscle and only a thin layer of subcutaneous tissue between the skin and the bone measuring approximately $3.8 \mathrm{~mm}$ (Cichowitz et al 2009). This subcutaneous tissue is attached to underlying deep fascia and the fibres of the Achilles tendon. The Achilles is the strongest and largest tendon in the body, but its suboptimal vascular supply and the high tensions it is subjected to make it prone to injuries including pressure ulcers (Cichowitz et al 2009).

\section{TIME OUT 2}

Do the risk assessments used in your clinical area include risk factors for pressure ulcers? What do you need to consider in your risk assessment to identify patients who are at high risk of developing pressure ulcers on the heel?

\section{Causal factors in the development of heel pressure ulcers}

Direct causal factors in the development of heel pressure ulcers include the duration and magnitude of any pressure exerted on the heel. The ability of soft tissues to tolerate the effects of pressure is influenced by intrinsic factors (those originating from within the body) such as malnutrition, diabetes mellitus and conditions that affect the circulation, and by extrinsic factors (those originating from outside of the body) such as shear, friction and moisture (Braden and Bergstrom 1987,
Defloor 1999). Other causal factors that can directly increase the vulnerability of the heel to pressure damage include immobility, the patient's skin status, the presence of previous pressure ulcers and/or scar tissue, and suboptimal tissue perfusion (Coleman et al 2014). While immobility is a significant risk factor for heel pressure ulcers, the nurse should always consider other causal factors which may act in combination with immobility to produce pressure ulcers on the heel.

\section{Immobility, shear and friction} Patients who spend significant periods of time in a supine position, for example those on extended bedrest, may find that their feet tend to abduct (rotate externally). This rotation causes pressure to pass through the lateral (outer) aspect of the heel to the calcaneus, where the fat pad is thin and irregularly shaped (Tenenbaum et al 2013). Patients who have undergone surgery are at increased risk of heel pressure ulcers because analgesia and anaesthesia reduce their pain responses to pressure and because they are immobilised for prolonged periods (Edwards et al 2006). Orthopaedic and vascular surgical interventions may require postoperative

immobilisation of the lower limb.

Shear and friction are common extrinsic factors increasing the risk of heel pressure ulcers. Shear occurs when the bones and the skin move in opposite directions - for example during suboptimal repositioning where the patient is pulled along the bed - and is a common issue in patients with suboptimal mobility (Davies 2018). Shear affecting the heels can occur if the patient slides downwards due to inadequate positioning in a chair or bed, or if the patient uses their heels as a pivot point when repositioning themselves. Friction can be described as the mechanical force exerted when a patient is moved across a coarse surface such as bed sheets, which prevents the skin from sliding along smoothly (Gleeson 2016). Friction to the heel can be caused by a suboptimal moving and handling technique or by frequent foot movements, for example in patients experiencing tremors or agitation. 
Skin status and presence of pressure ulcers

The presence of a category 1 pressure ulcer is correlated with a two-to-threefold increase in a patient's risk of developing a more severe pressure ulcer (Nixon et al 2007). The presence of scar tissue and the presence of dry, thin or oedematous skin also increase the pressure ulcer risk. Ageing has been found to increase the risk of pressure ulcers due to the thinning of the epidermis (outer layer of the skin) as part of the normal ageing process (Baumgarten et al 2006). Ageing also causes the skin to become dehydrated and lacking in sebum (Tong et al 2016), connective tissue to lose its elasticity and strength, and blood vessels to become increasingly fragile (Defloor 1999). Furthermore, subcutaneous tissues become thinner with age, which reduces the already minimal padding over the calcaneus and reduces the heel's capacity to absorb shock (Alcántara et al 2002).

\section{Suboptimal tissue perfusion}

The lower limbs are particularly vulnerable to reduced perfusion, which causes a reduction in the supply of oxygen and nutrients to the tissues in the foot. Suboptimal lower limb perfusion has been estimated to affect $50-62 \%$ of patients with a heel pressure ulcer (Meaume and Faucher 2008, McGinnis et al 2014b). Examples of conditions that can cause suboptimal tissue perfusion include cardiovascular disease or coronary artery disease, diabetes, infections, peripheral vascular disease, stroke and renal disease. Suboptimal tissue perfusion can also be caused by the effects of cigarette smoking, hypotension, hypoalbuminaemia and the use of inotropes (medicines that affect the strength of heart contractions) to support cardiac function (Coleman et al 2014, EPUAP et al 2019).

When a patient is acutely unwell, their sympathetic nervous system preserves their vital organs notably the heart and brain - by increasing circulation to these organs, which in turn results in decreased circulation to the peripheries. Vasopressors (medicines that induce vasoconstriction and raise blood pressure) work in a similar way. Therefore, acutely unwell patients such as those in intensive care settings are at higher risk than others of developing heel pressure ulcers (Coleman et al 2014, EPUAP et al 2019).

Peripheral vascular disease refers to any disease or disorder of the circulatory system outside the brain and heart. Peripheral vascular disease frequently affects the lower limbs and is caused by arteries becoming narrowed, weakened or blocked, resulting in reduced circulation to the feet. Peripheral vascular disease is commonly associated with older age but can also affect younger people who smoke or those with diabetes or hypertension (Tendera et al 2011).

Circulatory conditions such as peripheral vascular disease can lead to an increase in pedal (foot and ankle) oedema, which impairs the delivery of oxygen and nutrients to the tissues as well as the disposal of metabolic waste. Oedema can cause stiffening of the tissues, which affects pressure distribution, leading to greater stress during loading (when force and/or pressure is exerted through the tissues) (Gefen 2010). Oedematous lower limbs are also heavier, which can increase the pressure exerted on the heels.

\section{TIME OUT 3}

If a patient with diabetes develops an ulcer on their heel, how will you decide whether it is a diabetic foot ulcer or a pressure ulcer? Consider factors such as the patient's mobility, the exact location of the ulcer on the heel, the quality of perfusion to the heel area and whether the patient has any sensation in the foot

\section{Diabetes mellitus}

Extended periods of hyperglycaemia as a result of diabetes can cause a thickening and interlinking of collagen fibres in the connective tissues, leading to progressive tissue stiffening (Gefen 2010). Diabetes can also cause foot ulceration, primarily over pressure points such as the toes and the plantar foot, often caused by accidental trauma, ill-fitting footwear and/or peripheral neuropathy (Jeffcoate and Harding 2003). Peripheral neuropathy involves nerve damage to the peripheral nervous system

\section{Key points}

- The anatomy of the heel makes it highly vulnerable to pressure damage

- Heel pressure ulcers can cause pain, reduce mobility, prolong hospital stays and potentially result in amputation

- Risk factors for heel pressure ulcers include immobility, skin status, presence of previous pressure ulcers and/or scar tissue and suboptimal tissue perfusion

- Careful consideration of possible causes is needed to differentiate between a heel pressure ulcer and a diabetic foot ulcer located on the heel

- Optimal moving and handling, and regular repositioning are crucial for heel pressure ulcer prevention

- Patients at risk of heel pressure ulcers may benefit from medical devices designed to offload the heel, maintain a low pressure or reduce shear and friction

and can cause numbness as well as pain and weakness (Howarth 2019). Patients with peripheral neuropathy therefore become unaware of pain and pressure sensations that would normally indicate the need to reposition the foot and avoid trauma. As well as diabetes, peripheral neuropathy can also be caused by stroke, alcoholism, demyelinating diseases such as multiple sclerosis, and Guillain-Barré syndrome, a rare and serious condition that affects the nerves in the hands and feet (White et al 2004).

In patients with diabetes, it can be challenging for the nurse to differentiate between a diabetic foot ulcer located on the heel and a heel pressure ulcer. In this situation, the nurse needs to carefully consider the cause of the ulcer. For example, if the patient is immobile or bedbound and the ulcer affects the posterior aspect of the heel, it is likely to have been caused by pressure. Conversely, in a mobile patient or one whose ulcer is located on the plantar aspect of the heel, ill-fitting footwear may need to be considered as a potential cause. Regardless of the cause, any patient with diabetes 
FURTHER RESOURCES National Wound Care Strategy Programme -

Leg and Foot

Ulcers

www.nationa wound

carestrategy.

net/lower-limb

Legs Matter!

https://

legsmatter.org

Pressure Ulcer

Prevention and

Management

Resources

https://

tvs.org.uk/

pressure-ulcer-

prevention-

management

Helping

to Prevent

Pressure Ulcers:

A Quick Guide

for Registered

Managers of

Care Homes

www.nice.org.

uk/about/nice

communities/

social-care/

quick-guides/

helping-to-

prevent-

pressure-ulcers

Pressure Ulcers:

Applying All

Our Health

www.gov.uk/

government/

publications/

pressure-

ulcers-

applying-allour-health/

pressure-

ulcers-

applying-allour-health

\section{Permission}

To reuse this

article or for

information

about

reprints and

permissions,

contact

permissions@

reni.com and signs of foot ulceration should

be referred to a multidisciplinary

foot care service (National Institute

for Health and Care Excellence

(NICE) 2019).

\section{TIME OUT 4}

Think back to a bedbound patient you have cared for. When you repositioned the patient in bed, did you pay attention to how much their feet were moving during turning and to whether their heels were dragging along the bedsheets? What strategies did you use to reduce their risk of developing a heel pressure ulcer?

Prevention of heel pressure ulcers Because the development of a pressure ulcer is rarely due to a single causative factor, prevention strategies involve the nurse identifying each patient's individual risk factors, as described previously - for example, immobility, skin status, presence of pressure ulcers and/or scar tissue, and suboptimal tissue perfusion. This should involve a holistic risk assessment along with a thorough skin assessment. The signs of pressure damage that the nurse should look for when examining a patient's heels include any signs of redness, and whether the skin blanches (turns white when pressure is applied). It is not always possible to identify redness on darkly pigmented skin, so the nurse should assess for other signs of pressure damage including localised heat, oedema, blistering and induration (hardness) (Mitchell 2018).

In the UK, NICE states that patients in NHS secondary or longterm care must undergo a pressure ulcer risk assessment within six hours of admission (NICE 2014). If a patient is found to be at risk of pressure ulcers, a preventive care plan should be initiated.

An optimal moving and handling technique is crucial to prevent the development of pressure ulcers but its description is beyond the scope of this article. Another crucial component of any pressure ulcer preventive care plan is reducing the duration of pressure to the heels through regular repositioning. In some patients, however, regular repositioning may not be possible or may be insufficient to prevent the development of heel pressure ulcers. This is the case, for example, in some patients with peripheral arterial disease or those with immobilised limbs such as in lower limb fractures or those who may have been sedated in critical care. In these cases, the nurse may use their clinical judgement to decide that medical devices - such as support surfaces, heel-specific devices, pillows, wedges and prophylactic dressings - can be used to minimise the risk of heel pressure damage.

\section{TIME OUT 5}

Consider a patient you have cared for whose heels started to show signs of pressure damage. What techniques and devices did you use to prevent the development of a heel pressure ulcer? What was the rationale for your choice of device? Was your preventive care plan effective?

\section{Support surfaces}

The term support surface refers to mattresses, overlays and cushions. Support surfaces are generally divided into two categories: alternating pressure surfaces and constant low-pressure surfaces.

Alternating pressure devices work by reducing the duration of pressure exerted at the body's points of contact with the device. For example, alternating pressure mattresses use air-filled cells that inflate and deflate alternately during a set cyclical time period (usually every 5-10 minutes). Some alternating pressure mattresses contain specific 'heel zones' that feature an area of air-filled cells designed to reduce pressures at the heel.

Constant low-pressure devices distribute the body's weight over a larger surface area, reducing the pressure exerted on all body parts (McInnes et al 2015). They include foam mattresses, low air-loss mattresses and overlays.

There is little evidence regarding the effectiveness of support surfaces in preventing heel pressure ulcers specifically, but they are frequently used as part of a multifaceted care plan (Greenwood 2020).

\section{Heel-specific devices}

Any heel-specific device should increase blood flow to the heel area, reduce pressure and prevent shear. The device should be comfortable, practical - in that it can be used in all positions and during mobilisation - and cost-effective (Huber et al 2008). It should also minimise hyperextension of the knee, because this can compress the popliteal vein and contribute to the development of deep vein thrombosis (Huber and Huber 2009). There are several types of heel-specific devices, which can generally be categorised as:

》 Heel-offloading devices.

" Heel-specific constant lowpressure devices.

》 Heel-specific low-friction devices.

\section{Heel-offloading devices}

Heel-offloading devices work by maintaining surface contact with the foot and lower leg while 'floating' or suspending the heel. Heeloffloading devices vary in design and materials and can be single-use or re-usable. When used effectively, they can fully offload the heel and be used for up to 24 hours per day. Some designs reduce shear and friction and/or assist in preventing footdrop, where prolonged bedrest causes an over-stretching of the muscles and nerves and weakness in the lower limb. One meta-analysis demonstrated a reduction in heel pressure ulcer development with the use of offloading devices compared with standard care. However, further research is required because the quality of the available evidence was rated as low to moderate (Greenwood et al 2020a).

\section{Heel-specific constant low-} pressure devices

Heel-specific constant low-pressure devices reduce pressure to the heel by providing padding under the foot, thereby increasing the surface contact area. They include 'booties', heel cups or overlays that sit under the heel and can be made of foam, sheepskin, gel or other materials. There is little evidence of their clinical effectiveness (Greenwood et al 2020a). However, in the author's clinical experience, they can be beneficial in certain situations; for example, some heel-specific constant low-pressure devices can be used as orthotic insoles in those at risk of heel pressure damage or during lower-limb surgery and vascular radiology, during which limb stability is essential and the heel cannot be repositioned. 
Heel-specific low-friction devices In patients who find it challenging to contain movement in the lower limb, such as those experiencing tremors or agitation, the priority is to reduce shear and friction rather than pressure. In such cases, according to the author's clinical experience, foam dressings or 'booties' designed to reduce shear and friction can be more appropriate than offloading or constant low-pressure devices.

\section{Pillows and wedges}

In clinical practice, pillows and wedges are commonly used to offload pressure. Pillows and wedges are practical short-term methods that can be used in patients who are alert and co-operative and can maintain the same position (EPUAP et al 2019). They are less suited for more mobile patients, patients demonstrating signs of agitation and patients who frequently move the lower limb. They do not assist in preventing footdrop.

Pillows and wedges should be placed under the full length of the patient's calf, avoiding hyperextension of the knee, with the heel suspended above the mattress. If the pillow or wedge is placed inappropriately, the heel may still be in contact with a hard surface area of the bed. The thickness of pillows and wedges varies. If they are too thin or if the limb is too heavy, the heel may not be fully offloaded.

Figure 2 shows the appropriate heel elevation using a pillow.

\section{Prophylactic dressings}

Studies have demonstrated that foam dressings can reduce pressure as well as shear and friction (Levy et al 2015, Levy and Gefen 2016). Nakagami et al (2006) found that simple film dressings could limit the effects of shear, while according to a systematic review by Moore and Webster (2018) that investigated dressings and topical agents for preventing pressure ulcers, the incidence of pressure ulcers in all body areas was reduced with the use of silicone foam dressings. However, further evidence is required due to the suboptimal quality of some of the trials. In terms of the heel area specifically, there is no direct evidence of the effectiveness of any of these dressings (Greenwood et al 2020b).

\section{Homemade devices}

Historically, nurses have found resourceful solutions when seeking to relieve pressure on their patients' heels, often using homemade devices to prevent the development of pressure ulcers. These homemade solutions include using water-filled latex gloves to cushion the heel and intravenous fluid bags placed under the heel to increase elevation. However, it has been known for some time that water-filled gloves can lead to higher pressures at the heel, while intravenous fluid bags can increase the pressure at the Achilles tendon (Lockyer-Stevens 1993, Williams 1993). Therefore, while resourceful, these methods are not recommended.

\section{Implications for practice}

In terms of pressure ulcer prevention, the heel area requires specific attention and treatment because of its unique anatomy and its vulnerability to the effects of impaired peripheral circulation. The early identification of at-risk patients and signs of pressure damage followed by the initiation of a preventive care plan can reduce the risk and occurrence of pressure ulcers. All members of the nursing team are responsible for assessing the patient's skin, identifying signs of pressure damage, assessing it accurately and implementing the preventive measures discussed previously.

When caring for a patient at risk of heel pressure ulcers, the nurse can seek advice from the local tissue viability nurse specialist who will be able to provide guidance on appropriate medical devices. Tissue viability nurse specialists can also advise on patients who may be at high risk of developing heel pressure ulcers, such as patients who are immobile, patients with diabetes and/or patients who may not adhere to their treatment, as well as on measures to prevent heel pressure damage (Ousey et al 2014).

\section{Conclusion}

Heel pressure ulcers can have a significant negative effect on a patient's health-related quality of life, causing pain, reducing mobility, prolonging hospital stays, and in severe cases resulting in amputation. Techniques and devices, if appropriately selected and implemented, can reduce the effects of pressure, shear and friction on patients' heels and prevent the development of heel pressure ulcers, thereby helping to maintain patients' quality of life. Therefore, it is important that nurses understand how and why pressure damage may develop on the heel, how to assess the risk of heel pressure ulcers, and which preventive measures they should implement.

\section{TIME OUT 6}

Consider how understanding the development and prevention of heel pressure ulcers relates to the Code: Professional Standards of Practice and Behaviour for Nurses, Midwives and Nursing Associates (Nursing and Midwifery Council 2018) or, for non-UK readers, the requirements of your regulatory body

\section{TIME OUT 7}

Now that you have completed the article, reflect on your practice in this area and consider writing a reflective account: rcni.com/reflective-account
Figure 2. Appropriate heel elevation using a pillow
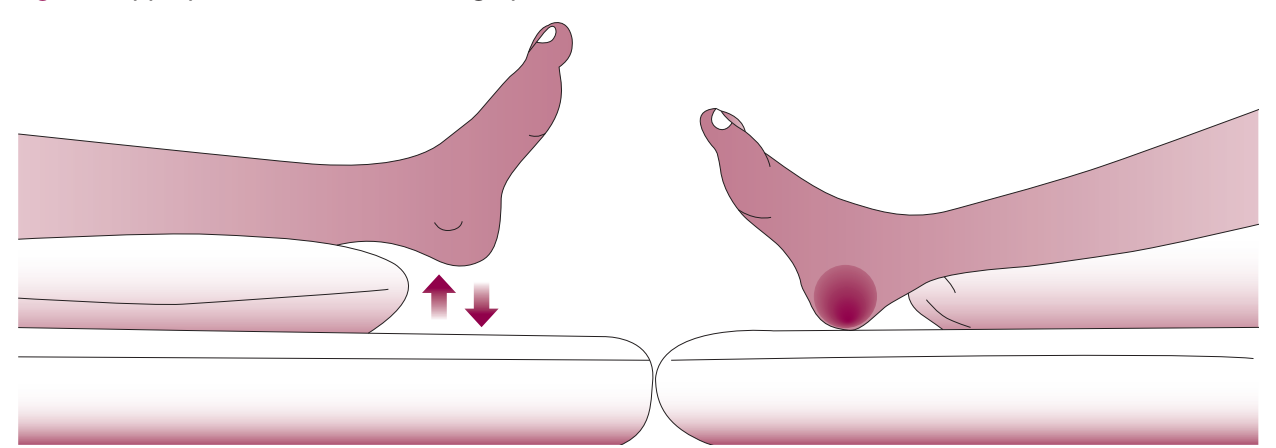

Martin Faulks 


\section{References}

Alcántara E, Forner A, Ferrús E et al (2002) Influence of age, gender, and obesity on the mechanical properties of the heel pad under walking impact conditions. Journa of Applied Biomechanics. 18, 4, 345-356. doi: 10.1123/jab.18.4.345

Baumgarten M, Margolis DJ, Localio AR et al (2006) Pressure ulcers among elderly patients early in the hospital stay. The Journals of Gerontology Series A: Biological Sciences and Medical Sciences. 61, 7,749-754. doi: 10.1093/gerona/61.7.749

Braden B, Bergstrom N (1987) A conceptual schema for the study of the etiology of pressure sores. Rehabilitation Nursing Journal. 12, 1, 8-12. doi: 10.1002/j.2048-7940.1987.tb00541.x

Briggs M, Collinson M, Wilson Let al (2013) The prevalence of pain at pressure areas and pressure ulcers in hospitalised patients. BMC Nursing. 12, 19. doi: 10.1186/1472-6955-12-19

Cichowitz A, Pan WR, Ashton M (2009) The heel: anatomy, blood supply, and the pathophysiology of pressure ulcers. Annals of Plastic Surgery. 62, 4, 423-429. doi: 10.1097/SAP.0b013e3181851b55

Coleman S, Nixon J, Keen J et al (2014) A new pressure ulcer conceptual framework. Journal of Advanced Nursing. 70, 10, 2222-2234. doi: 10.1111/jan.12405

Davies $\mathrm{P}$ (2018) Preventing the development of heel pressure ulcers. Nursing Standard. doi: 10.7748/ns.2018.e11294

Defloor T (1999) The risk of pressure sores: a conceptual scheme. Journal of Clinical Nursing 8, 2, 206-216. doi: 10.1046/j.1365-2702.1999.00254.x

Edwards JL, Pandit H, Popat MT (2006) Perioperative analgesia: a factor in the development of heel pressure ulcers? British Journal of Nursing. 15, 6 (Suppl) S20-S25 doi: 10.12968/bjon.2006.15.Sup1.20688

European Pressure Ulcer Advisory Panel, National Pressure Injury Advisory Panel, Pan Pacific Pressure Injury Alliance (2019) Prevention and Treatment of Pressure Ulcers/Injuries: Clinical Practice Guideline. The International Guideline EPUAP, NPIAP, PPPIA.

Fowler E, Scott-Williams S, McGuire JB (2008) Practice recommendations for preventing hee pressure ulcers. Ostomy/Wound Management. $54,42-48,50-52,54-57$.
Gefen A (2010) The biomechanics of heel ulcers. Journal of Tissue Viability. 19, 4, 124-131. doi: 10.1016/j.jtv.2010.06.003

Gleeson D (2016) Heel pressure ulcer prevention: a 5-year initiative using low-friction bootees in a hospital setting. Wounds UK, 12, 4, 80-87.

Gorecki C, Brown JM, Nelson EA et al (2009) Impact of pressure ulcers on quality of life in older patients: a systematic review. Journal of the American Geriatrics Society. 57, 7, 1175-1183. doi: 10.1111/j.1532-5415.2009.02307.x

Greenwood C (2020) An exploration of the use of devices for the prevention of heel pressure ulcers in secondary care: a realist evaluation. Doctoral thesis, University of Leeds, Leeds, England.

Greenwood C, Nelson A, Nixon J et al (2020a) Heel Specific Devices for the Prevention of Hee Pressure Ulcers - A Systematic Review. European Wound Management Association, virtual conference, 19 November 2020.

Greenwood C, Nelson A, Nixon J et al (2020b) Prophylactic dressings for the prevention of heel pressure ulcers. European Pressure Ulcer Advisory Panel, virtual conference, 24 September 2020.

Guest JF, Ayoub N, Mcilwraith T et al (2015) Health economic burden that wounds impose on the National Health Service in the UK. BMJ Open. 5, e009283. doi: 10.1136/bmjopen-2015-009283

Howarth D (2019) Preventing foot complications in people with diabetes mellitus. Nursing Standard. doi: 10.7748/ns.2019.e11432

Huber DE, Huber JP (2009) Popliteal vein compression under general anaesthesia. European Journal of Vascular and Endovascular Surgery. 37, 4, 464-469. doi: 10.1016/j.ejvs.2008.11.015

Huber JP, Reddy R, Pitham T et al (2008) Increasing heel skin perfusion by elevation. Advances in Skin and Wound Care. 21, 1, 37-41. doi: 10.1097/01.ASW.0000284968.07982.26

Jeffcoate WJ, Harding KG (2003) Diabetic foot ulcers. The Lancet. 361, 9368, 1545-1551. doi: 10.1016/S0140-6736(03)13169-8

Levy A, Frank MB, Gefen A (2015) The biomechanical efficacy of dressings in preventing heel ulcers. Journal of Tissue Viability. 24, 1, 1-11. doi: 10.1016/j.jtv.2015.01.001
Levy A, Gefen A (2016) Computer modeling studies to assess whether a prophylactic dressing reduces the risk for deep tissue injury in the heels of supine patients with diabetes. 0stomy/Wound Management. 62, 4, 42-52.

Lockyer-Stevens N (1993) The use of water-filled gloves to prevent the formation of decubitus ulcers on heels. Journal of Wound Care. 2, 5, 282-285. doi: 10.12968/jowc.1993.2.5.282

McGinnis E, Briggs M, Collinson M et al (2014a) Pressure ulcer related pain in community populations: a prevalence survey. BMC Nursing 13. 16. doi: 10.1186/1472-6955-13-16

McGinnis E, Greenwood DC, Nelson EA et al (2014b) A prospective cohort study of prognostic factors for the healing of heel pressure ulcers. Age and Ageing. 43, 2, 267-271 doi: 10.1093/ageing/aft187

McInnes E, Jammali-Blasi A, Bell-Syer SE et al (2015) Support surfaces for pressure ulcer prevention. Cochrane Database of Systematic Reviews. 9, CD001735. doi: 10.1002/14651858.CD001735

Meaume S, Faucher N (2008) Heel pressure ulcers on the increase? Epidemiologica change or ineffective prevention strategies? Journal of Tissue Viability. 17, 1, 30-33. doi: 10.1016/j.jtv.2007.09.010

Mitchell A (2018) Adult pressure area care: preventing pressure ulcers. Britis Journal of Nursing. 27, 18, 1050-1052. doi: 10.12968/bjon.2018.27181050

Moore ZE, Webster J (2018) Dressings and topical agents for preventing pressure ulcers. Cochrane Database of Systematic Reviews. 12, CD009362. doi: $10.1002 / 14651858$

Nakagami G, Sanada H, Konya C et al (2006) Comparison of two pressure ulcer preventive dressings for reducing shear force on the heel. Journal of Wound Ostomy and Continence Nursing. 33, 3, 267-272. doi: 10.1097/00152192-200605000-00007

National Institute for Health and Care Excellence (2014) Pressure Ulcers: Prevention and Management. Clinical guideline No. 179. NICE, London.

National Institute for Health and Care Excellence (2019) Diabetic Foot Problems: Prevention and
Management. NICE guideline No. 19. NICE, London.

Nixon J, Cranny G, Bond S (2007) Skin alterations of intact skin and risk factors associated with pressure ulcer development in surgical patients: a cohort study. International Journal of Nursing Studies. 44, 5, 655-663. doi: 10.1016/j.jinurstu.2006.02.010

Nursing and Midwifery Council (2018) The Code: Professional Standards of Practice and Behaviour for Nurses, Midwives and Nursing Associates. NMC, London.

Ousey K, Atkin L, Milne J et al (2014) The changing role of the tissue viability nurse: an exploration of this multifaceted post. Wounds UK. 10, 4, 54-61.

Spilsbury K, Nelson A, Cullum N et al (2007) Pressure ulcers and their treatment and effects on quality of life: hospital inpatient perspectives. Journal of Advanced Nursing. 57, 494-504. doi: 10.1111/j.1365-2648.2006.04140.x

Tendera M, Aboyans V, Bartelink Ml et al (2011) ESC Guidelines on the diagnosis and treatment of peripheral artery diseases: document covering atherosclerotic disease of extracranial carotid and vertebral, mesenteric, renal, upper and lower extremity arteries: the Task Force on the Diagnosis and Treatment of Peripheral Artery Diseases of the European Society of Cardiology (ESC). European Heart Journal. 32, 22, 2851-2906 doi: 10.1093/eurheartj/ehr21

Tenenbaum S, Shabshin N, Levy A et al (2013) Effects of foot posture and heel padding devices on soft tissue deformations under the heel in supine position in males: MRI studies. Journa of Rehabilitation Research and Development. 50, 8, 1149-1156. doi: 10.1682/JRRD/2012.10.0183

Tong Sf, Yip J, Yick KL et al (2016) Effects of different heel angles in sleep mode on heel interface pressure in the elderly. Clinical Biomechanics. 32, 229- 235 doi: 10.1016/j.clinbiomech.2015.11.006

White CM, Pritchard J, Turner-Stokes L (2004) Exercise for people with peripheral neuropathy. Cochrane Database of Systematic Reviews. 4, CD003904. doi: 10.1002/14651858.CD003904.pub2

Williams C (1993) Using water-filled gloves for pressure relief on heels. Journal of Wound Care 2, 6, 345-348. doi: 10.12968/jowc.1993.2.6.345 


\section{Heel pressure ulcers}

1. In the past, pressure ulcers were also known as:

a) Venous leg ulcers

b) Decubitus ulcers

c) Diabetic foot ulcers

d) Duodenal ulcers

2. Which of the following is a potential consequence of serious infection secondary to pressure ulcers?

a) Peripheral vascular disease

b) Coma

c) Blood transfusion

d) Amputation

3. Pressure ulcers are categorised according to:

a) Extent of bruising

b) Odour and skin type

c) Depth and severity

d) Bleeding risk

4. Which of the following pressure ulcer categories is described as 'intact skin with a localised area of non-blanching erythema'?
a) Category 1
b) Category 4
c) Unstageable pressure ulcer
d) Suspected deep tissue injury

5. The heel is often reported as the second most common site for the development of pressure ulcers after which other area?

a) Buttocks

b) Knees

c) Elbows

d) Sacrum

6. What is the approximate width of the layer of subcutaneous tissue at the back of the heel?
a) One inch
b) $5 \mathrm{~mm}$
c) $3.8 \mathrm{~mm}$
d) $1.5 \mathrm{~cm}$

7. Which of the following causal factors can directly increase the vulnerability of the heel to pressure damage?

a) Immobility

b) Skin status

c) Suboptimal tissue perfusion

d) All of the above

8. Friction can be described as:

a) The effect of the bones and the skin moving in opposite directions

b) The mechanical force exerted when a patient is moved across a coarse surface such as bed linen

c) The effect of compression caused by dressings and bandaging

d) The pressure exerted by fixed medical devices such as tubes and catheters

9. Ageing has been found to increase the risk of pressure ulcers due to:

a) Loss of melatonin in the skin

b) Weakening bone strength

c) Thinning of the epidermis

d) Reduced eyesight

10. Strategies that can be used to prevent heel pressure ulcers include:

a) Optimal handling and moving technique

b) Regular repositioning

c) The use of medical devices such as mattresses, cushions and dressings

d) All of the above
How to complete

this quiz

This multiple-choice quiz will help you to test your knowledge. It comprises ten questions that are broadly linked to the CPD article.

There is one correct answer to each question.

" You can test your subject knowledge by attempting the questions before reading the article, and then go back over them to see if you would answer any differently. "You might like to read the article before trying the questions.

Subscribers making use of their RCNi Portfolio can complete this and other quizzes online and save the result automatically. Alternatively, you can cut out this page and add it to your professional portfolio. Don't forget to record the amount of time taken to complete it.

Further multiple-choice quizzes are available at rcni.com/cpd/test-yourknowledge

This multiple-choice quiz was compiled by Jason Beckford-Ball

The answers to this multiplechoice quiz are:

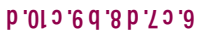

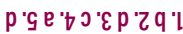

This activity has taken me _ minutes/hours to complete. Now that I have read this article and completed this assessment, I think my knowledge is: 